\title{
The MANIFEST fibre positioning system for the Giant Magellan Telescope
}

Jon S. Lawrence*a, David M. Brown ${ }^{\mathrm{a}}$, Jurek Brzeski ${ }^{\mathrm{a}}$, Scott Case ${ }^{\mathrm{a}}$, Matthew Colless ${ }^{\mathrm{b}}$, Tony Farrell ${ }^{\mathrm{a}}$, Luke Gers $^{\mathrm{a}}$, James Gilbert ${ }^{\mathrm{a}}$, Michael Goodwin ${ }^{\mathrm{a}}$, George Jacoby ${ }^{\mathrm{c}}$, Andrew M. Hopkins ${ }^{\mathrm{a}}$, Michael Ireland ${ }^{\mathrm{a}}$, Kyler Kuehn ${ }^{\mathrm{a}}$, Nuria P. F. Lorente ${ }^{\mathrm{a}}$, Stan Miziarski ${ }^{\mathrm{a}}$, Rolf Muller ${ }^{\mathrm{a}}$, Vijay Nichani ${ }^{\mathrm{a}}$, Azizi Rakman ${ }^{\mathrm{a}}$, Samuel Richards ${ }^{\mathrm{d}}$, Will Saunders ${ }^{\mathrm{a}}$, Nick F. Staszak ${ }^{\mathrm{a}}$, Julia Tims ${ }^{\mathrm{a}}$, Minh Vuong ${ }^{\mathrm{a}}$, Lew Waller ${ }^{\mathrm{a}}$

${ }^{a}$ Australian Astronomical Observatory, North Ryde, NSW 2113, Australia;

${ }^{b}$ Research School of Astronomy \& Astrophysics, The Australian National University, Mount Stromlo Observatory, ACT 2611, Australia;

${ }^{c}$ Giant Magellan Telescope/Carnegie Observatories, 813 Santa Barbara Street, Pasadena, CA 91101, USA;

${ }^{\mathrm{d}}$ School of Physics, The University of Sydney, NSW 2006, Australia

\begin{abstract}
MANIFEST is a fibre feed system for the Giant Magellan Telescope that, coupled to the seeing-limited instruments GMACS and G-CLEF, offers qualitative and quantitative gains over each instrument's native capabilities in terms of multiplex, field of view, and resolution. The MANIFEST instrument concept is based on a system of semi-autonomous probes called "Starbugs" that hold and position hundreds of optical fibre IFUs under a glass field plate placed at the GMT Cassegrain focal plane. The Starbug probes feature co-axial piezoceramic tubes that, via the application of appropriate AC waveforms, contract or bend, providing a discrete stepping motion. Simultaneous positioning of all Starbugs is achieved via a closed-loop metrology system.
\end{abstract}

Keywords: fibre positioner, Starbugs, fibre robots, GMT

\section{INTRODUCTION}

MANIFEST is a versatile and capable fibre feed and positioner [1,2] for all of GMT's natural-seeing or GLAO-fed spectrographs $[3,4]$. The baseline design for MANIFEST has 500 fibre-positioning 'Starbugs' that operate in parallel. The Starbugs have various geometries: single-aperture, image-slicing, or integral field unit (IFU); there are also fixed IFUs. MANIFEST's design is modular, connectorised, and extendable; it makes extensive use of tested technologies.

MANIFEST offers enhanced functionality for GMT spectrographs by providing:

- $\quad$ increased fields of view for all instruments;

- $\quad$ increased multiplex capability for all instruments;

- multiple deployable IFUs in a variety of sizes;

- an increased spectral resolution (factor 3-8) via image-slicing;

- an efficient detector packing, both spectrally and spatially;

- the capability to feed narrow spectrograph fields from fibres with a wide field;

- $\quad$ simultaneous observations with multiple instruments;

- the capability of feeding spectrographs at gravity-invariant locations;

- $\quad$ OH sky-line suppression in the near-infrared.

*j1@aao.gov.au; phone +61 29372 4853; fax +61 29372 4880; www.aao.gov.au

Ground-based and Airborne Instrumentation for Astronomy V, edited by Suzanne K. Ramsay, lan S. McLean, Hideki Takami, Proc. of SPIE Vol. 9147, 914794 - (C) 2014 SPIE · CCC code: 0277-786X/14/\$18 - doi: 10.1117/12.2055742

Proc. of SPIE Vol. 9147 914794-1 


\section{INSTRUMENT CONCEPT}

The overall instrument concept (see Figures 1 and 2) is similar to that presented in [1,2]. Starbug robots are held with a vacuum force underneath a glass field plate located at the telescope focal plane. A metrology camera located on the secondary mirror support structure will view back-illuminated fibres inside each Starbug to allow for closed-loop positioning. The field plate assembly includes the glass field plate and a connector plate that sits beneath a field flattening lens. The connector plate distributes electric power, vacuum hoses, science fibres, and metrology fibres to each of the Starbugs from common manifolds or the science instruments. The focal plane assembly fits within a sub-frame that can be installed and removed from the telescope focal plane via rails. When not in use the instrument is stored within a quadrant of the GMT Gregorian Instrument Rotator.

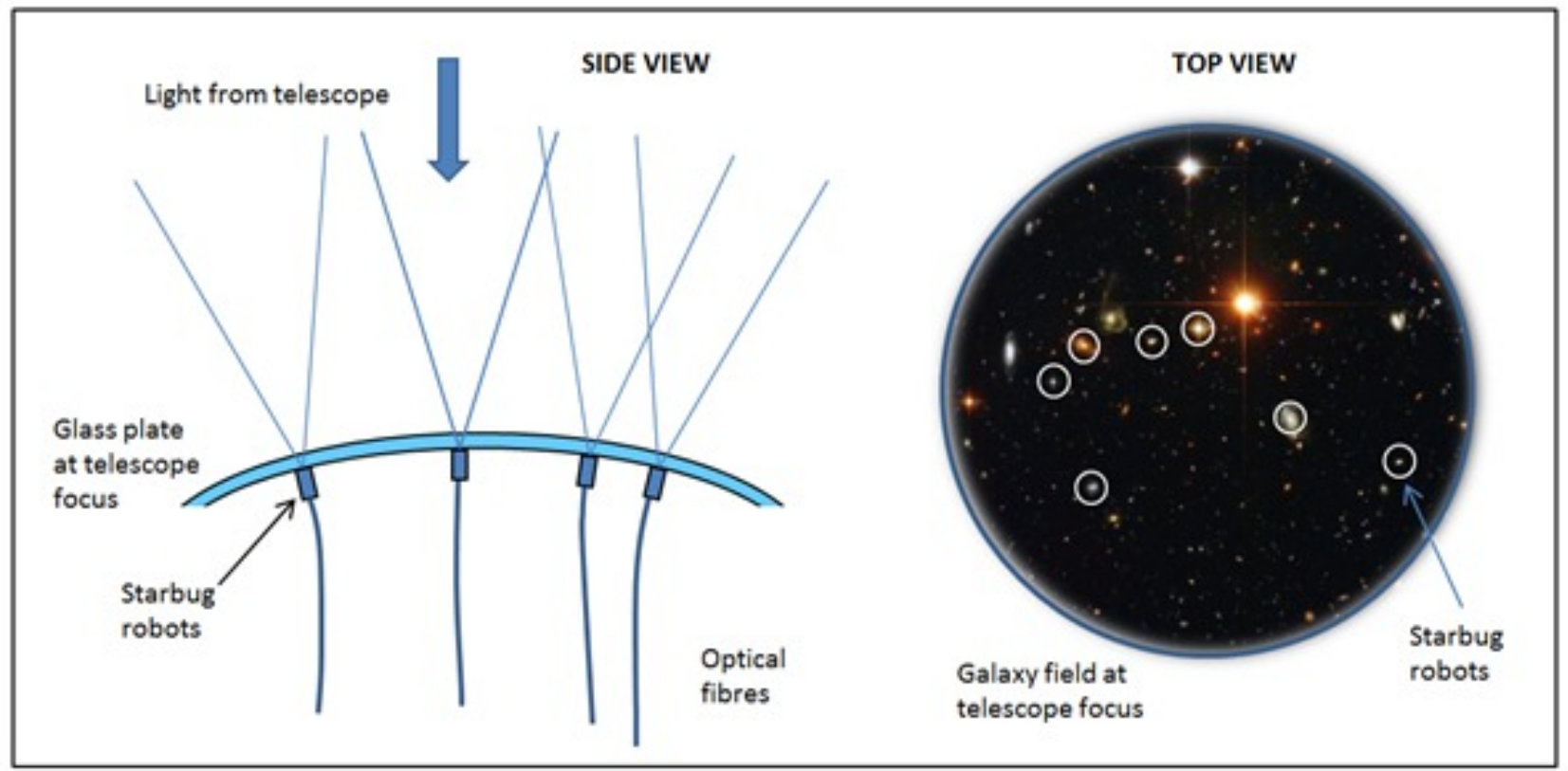

Figure 1. Concept for Starbugs operation in the telescope focal plane.
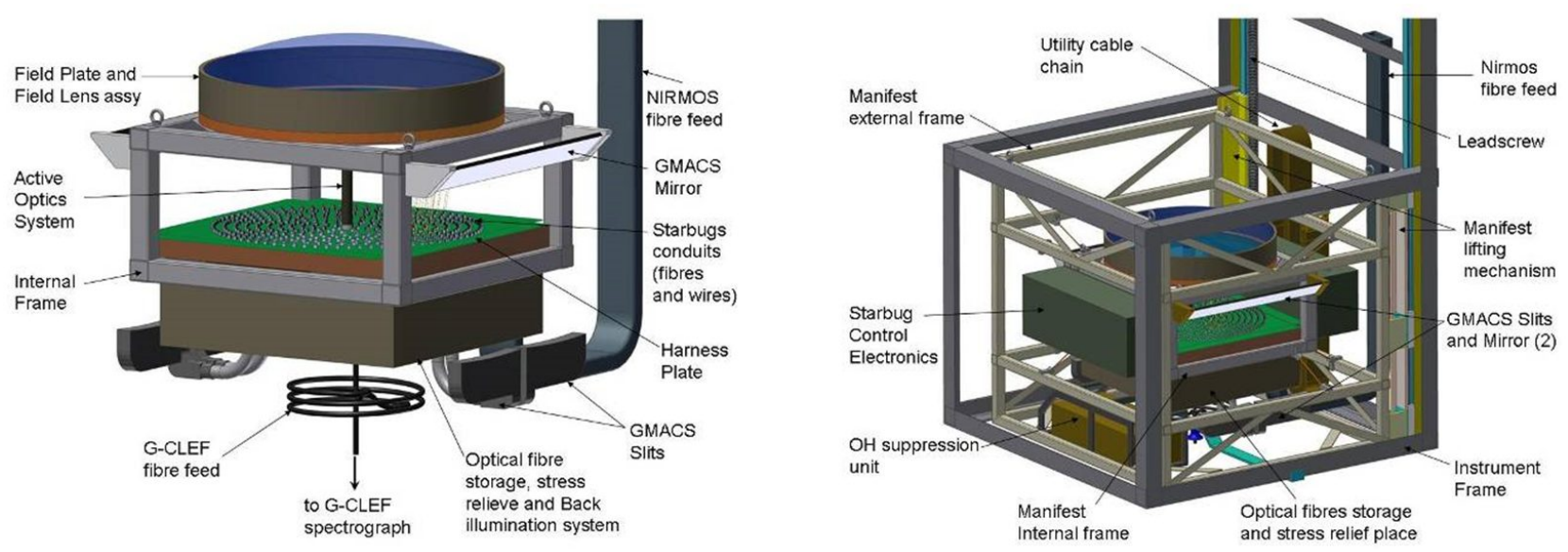

Figure 2. MANIFEST - internal (left) and external (right) frame sub-systems. 


\section{PROTOTYPING DESIGN STUDY}

We are currently conducting a Prototyping Design Study for MANIFEST. The aim for this project phase is to reduce technical risk for the instrument by improving the technological readiness. There are three primary objectives:

1. To continue R\&D work on the three key instrument subsystems (Starbugs robots, glass field plate, and the fibre system). The long-term motivation for this development is to increase instrument throughput and operating efficiency, and to reduce costs and technical risks for the eventual instrument subsystem elements.

2. To develop a working prototype of a Starbugs system on an astronomical telescope. The prototype system will operate on the UK Schmidt Telescope (UKST) operated by the AAO. We intend to augment this fibrepositioning prototype with upgrades to the telescope control system and spectrograph to provide a sciencecapable instrument. The operation of this instrument will explore and mitigate many system-level risks for MANIFEST.

3. To continue development of the MANIFEST concept. We are working with selected first-generation instrument teams (G-CLEF and GMACS), and with the GMTO, to ensure that the MANIFEST concept is optimal and consistent with instrument and telescope designs and interfaces.

\section{SUBSYSTEM RESEARCH AND DEVELOPMENT}

\subsection{Starbugs}

Starbug robots are the key technology for the MANIFEST instrument $[5,6,7]$. This Starbug subsystem has been developed in a number of areas [8] since the Feasibility Study for the instrument that was completed in 2011. Improvements have been made to the Starbug mechanical reliability and construction repeatability, the optical payload interface, the Starbug connectorisation, the electronics processes, and the Stabugs movement ability.

Mechanical reliability and construction repeatability has been improved by a number of design features. The piezo tube electrodes have been redesigned without either the inner or outer electrode at the joint to ensure there is no piezoelectric effect and no stress on the joint. The joint is increased in strength by replacing the collar with a potted joint of low shrinkage and low outgassing epoxy. Potting is achieved by a 3D printed formwork that aligns the tubes and metrology ferrules. It also contains a vacuum throttling hole. Soldering can then be achieved more reliably before assembly.

Optical Payloads have been incorporated into the Starbug design (see Figure 3) including metrology fibres, science fibres, and guide bundles. Three metrology fibres are installed between the two ceramic tubes to the upper joint. The astronomical science fibre is mounted in an off-the-shelf ceramic ferrule with its associated connection sleeve. It is polished partially in-situ and then installed (to within $50 \mu \mathrm{m}$ in the focus direction) using an alignment jig. The same mating sleeve has been used to hold a guide bundle (a $\sim 2 \mathrm{~mm}$ diameter polymer fibre) that can provide for object acquisition, telescope guiding, field plate focus, and field plate tip-tilt adjustment for the instrument. Further work in the next MANIFEST project phase will be required to implement multi-fibre IFU payloads.

A new design has been developed using a single connector for all services on each Starbug. This connector is a commercial $15 \mathrm{~mm}$ diameter hybrid optical fibre/electrical connector that has been modified to accommodate a vacuum feedthrough (see Figure 3). All electrical wires and the science fibre are routed through the vacuum tube that joins the Starbug to the connector. An LED for the metrology fibre sources can be mounted either on the Starbug itself or in the connector housing. This single unit will significantly enhance the instrument robustness and allow much easier replacement and maintenance of Starbugs within the instrument. The extension of this design to allow multi-sciencefibre connectors will occur in the next MANIFEST project phase.

Substantial progress has been made against the goal of optimizing the Starbugs electronics processes in order to reduce the size, weight, heat dissipation, and cost of the electronics modules. Modification to waveforms, the electrode configuration, and new actuators have lead to improvements in peak current (reduced by $40 \%$ ), amplifier capacity (increased by $50 \%$ ), power consumption (reduced by $23 \%$ ), nonlinearity (reduced from $14 \%$ to $1 \%$ ), and actuator sensitivity (increased by $25 \%$ ).

A method of rotating a Starbug about its centre has been developed allowing optimised routing and collision avoidance [9] with lower reconfiguration times, precise angular placement of non-symmetric payload, and field rotation 
compensation and tracking during observations. This is achieved through the addition of an electrode (a seventh) and a minor modification to the drive waveforms. This mode is now considered to be the baseline design.
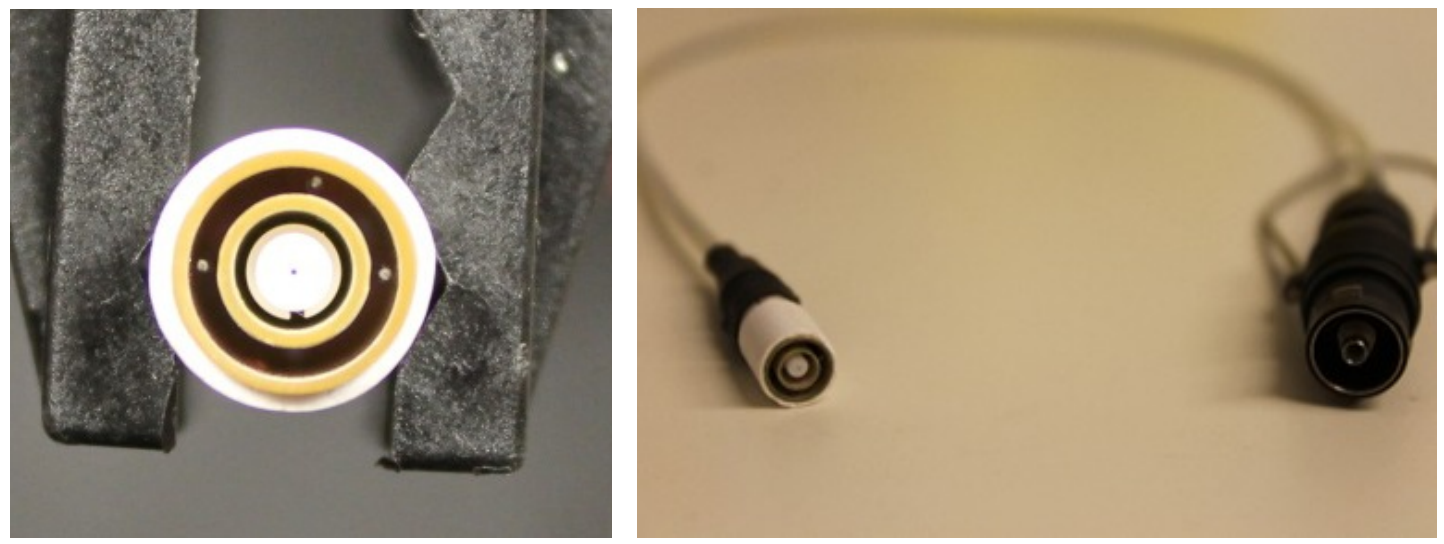

Figure 3. Left: Close-up view of Starbug "feet" showing science fibre mounted in central ferrule with three metrology fibres mounted between the Starbug tubes. Right: Starbug unit consisting of Starbug at one end and single connector at the other.

\subsection{Glass Field Plate}

The glass field plate for MANIFEST, being $1.3 \mathrm{~m}$ in diameter and $\sim 5 \mathrm{~mm}$ thick, is a challenging unit for fabrication and coating. Progress since the initial Feasibility Study has included a more detailed tolerance analysis and further communication with a number of potential vendors, both for the fabrication and the coating of the glass field plate.

We engaged a company to produce a $1.3 \mathrm{~m}$ wide field plate fabrication feasibility study. This study covered blank procurement and fabrication method, field plate optical fabrication, optical testing, shell handling, and packing/shipping, but excluded cost estimation. The fabrication method entails generating (and potentially slumping), grinding, and polishing of a blank. The study did not identify any major technical issues with the field plate fabrication though suggested further prototyping efforts would be useful. Other vendors of large glass lenses have indicated a preference for using a similar fabrication method. We have also procured a $400 \mathrm{~mm}$ diameter prototype plano-plano blank to test the slumping procedure.

We have also identified a number of coating suppliers for the field plate, who have provided broadband anti-reflection coating designs that meet specification. A witness sample for one of these coatings has been fabricated (see Figure 4) and tested by the AAO for spectral transmission, surface roughness and durability. Some issues where identified with the durability of this coating, however, and further samples are being procured.

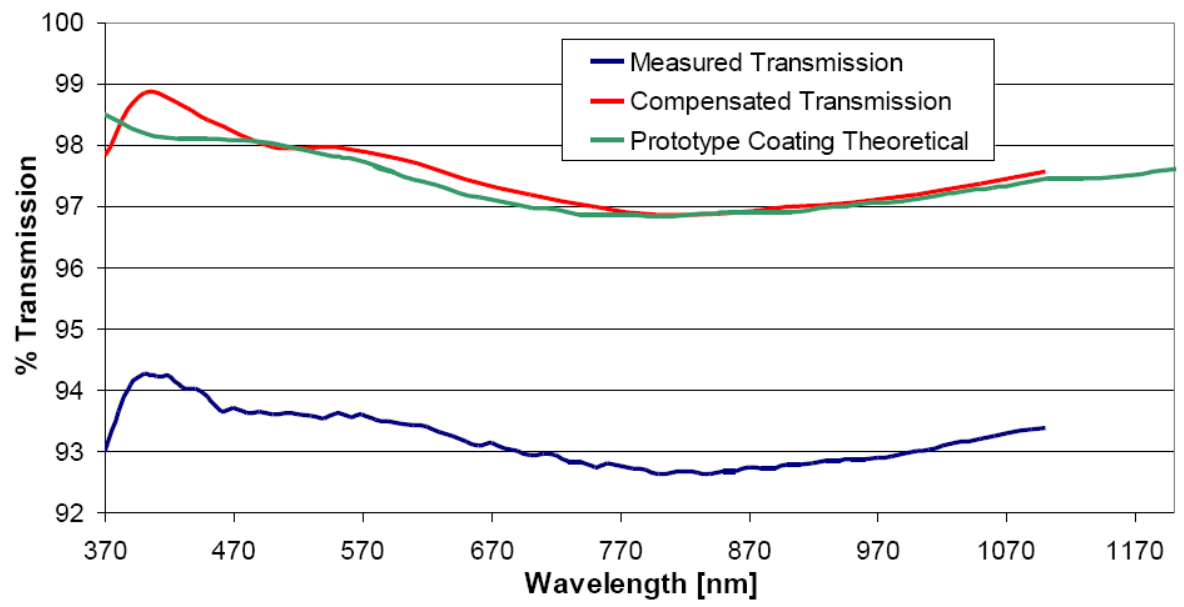

Figure 4. Transmission of witness sample (single side coating); there is good agreement between the theoretical transmission of the coating design and the measured transmission (when compensated for one surface loss and glass absorption). 


\subsection{Fibre Subsystem}

The fibre subsystem for MANIFEST consists of fore-optics mounted within each Starbug that feed an optical fibre bundle to post-optics mounted within each spectrograph. The fore-optics design comprises an initial hexagonal microlens array, followed by a circular microlens array, and then the fibre face. The post-optics for each Starbug reverse the input optics and use linear arrays to line the fibres with the spectrograph slit. The number of fibres within each Starbug input array is matched to the instrument and science case. Configurations have been proposed that have from 1 to 91 fibres, with the most common having 7 or 19 fibres. Larger wide field units are also possible with several thousand fibres, though these would be mounted in fixed units rather than deployable Starbugs.

Progress on the fibre subsystem for MANIFEST has been substantially enhanced through other AAO projects that have overlapping requirements. These projects include: KOALA [10], a 1000-element fibre IFU that feeds the AAOmega spectrograph at the AAT; SAMI [11], a multi hexabundle-IFU instrument that feeds AAOmega at the AAT, and GHOST [12], a high resolution image-slicing spectrograph for the Gemini telescope.

Work on the MANIFEST fibre subsystem components has consisted of developing the prescription for the key components, identifying suppliers who are capable of delivering these components to the required specification, and procuring and testing these components. Components have included the first and second microlens array pairs, the fibre bonded array, and the protected fibre bundle.

Three manufacturers of hexagonal microlens arrays have provided samples that have been tested and found to meet expectations in terms of scattered light. Results are shown in Table 1. Additionally, a prototype assembly that consists of an array of fibres bonded to a microlens array pair has been procured and tested. This was found to meet specification (see Figure 5). A large scale version of this assembly was then used in the KOALA instrument, now commissioned. The focal ratio degradation and absorption properties for a series of fibres of different type and manufacturer have been tested (Figure 6). Suitable fibre configurations and suppliers have been identified for the optical instruments of GMT. Tests on focal ratio degradation properties of various types of furcation tube and fibre packing density have also been performed. This study will inform the final specification for the MANIFEST fibre bundles.

Table 1. Measured characteristics of hexagonal lenslet arrays from three manufacturers.

\begin{tabular}{|l|l|l|l|c|}
\hline Manufacturer & Lenslet pitch & Fill factor & Relative cost & Comments \\
\hline SUSS & $249.7 \pm 0.1 \mu \mathrm{m}$ & $92 \%$ & Low & $\begin{array}{c}\text { Unacceptable } \\
\text { performance }\end{array}$ \\
\hline Fraunhofer IOF & $250.3 \pm 0.1 \mu \mathrm{m}$ & $94-96 \%$ & Medium & $\begin{array}{c}\text { Acceptable } \\
\text { performance }\end{array}$ \\
\hline Jenoptik & $250.2 \pm 0.1 \mu \mathrm{m}$ & $97-100 \%$ & High & $\begin{array}{c}\text { Acceptable } \\
\text { performance }\end{array}$ \\
\hline
\end{tabular}

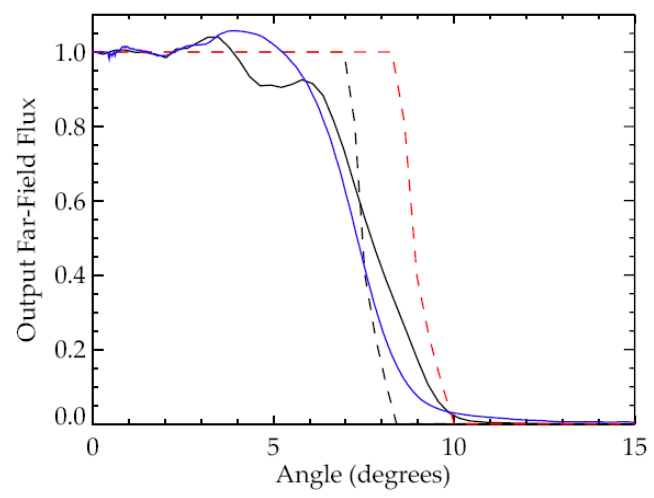

Figure 5. Focal ratio degradation analysis of the full dual-microlens injection prototype. The input far-field (black dashed line), the measured far-field output (black solid line), the far-field output determined from mutli-angle injection (blue solid line), and the weighting function of the hexagon on output (red dashed line) are shown. These results give acceptable performance for the prototype in terms of focal ratio degradation. 

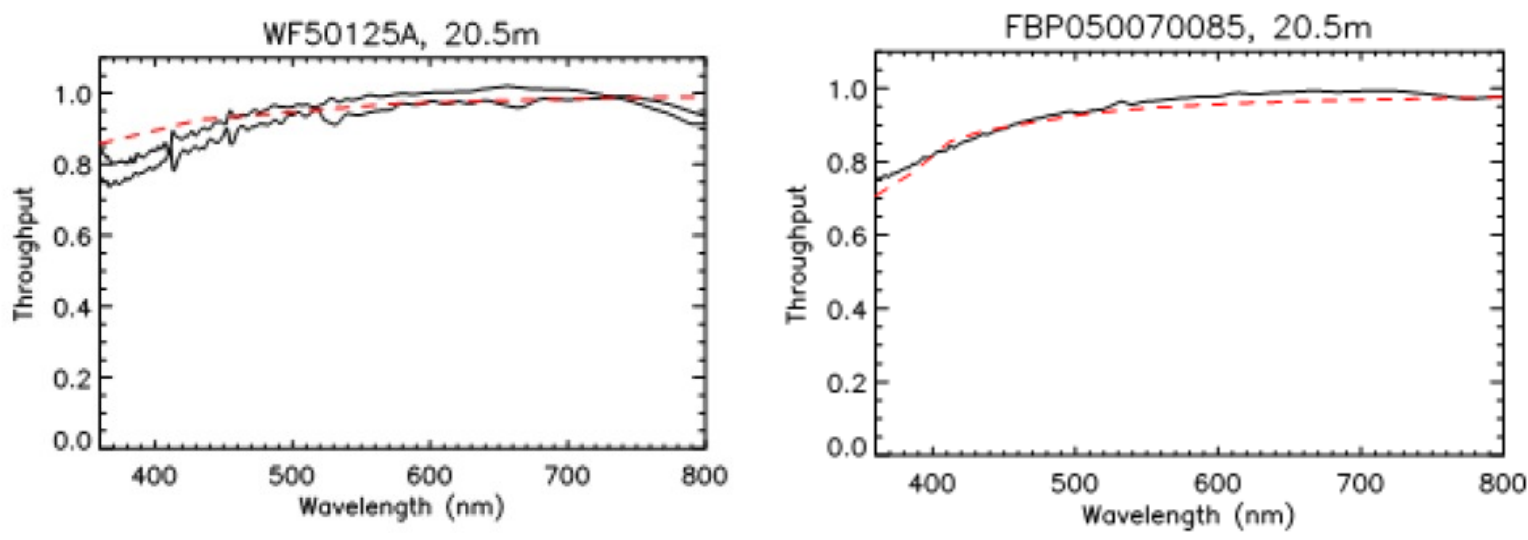

Figure 6. Throughput measurements of several different fibre types. Left: Optran WF from Ceramoptec with $50 \mu \mathrm{m}$ core and $125 \mu \mathrm{m}$ cladding. Right: FBP from Polymicro with $50 \mu \mathrm{m}$ core and $70 \mu \mathrm{m}$ cladding. In both plots the black curves are the experimental data compared to the manufacturer's specification.

\section{PROTOTYPING}

\subsection{On-telescope tests}

During early 2014, a series of Starbug prototyping experiments (see Figure 7) were performed on the United Kingdom Schmidt Telescope (UKST) at Siding Spring Observatory in northern New South Wales, Australia. These experiments were intended to reduce risk by proving some key concepts and verifying interfaces for the TAIPAN instrument. Experiments have included field plate focus and tip-tilt control using distributed coherent acquisition bundles, telescope guiding using a coherent bundle at field plate centre, metrology system mounting and flexure, field plate registration using fiducial fibres, and Starbug closed-loop control using the prototype metrology system [13].
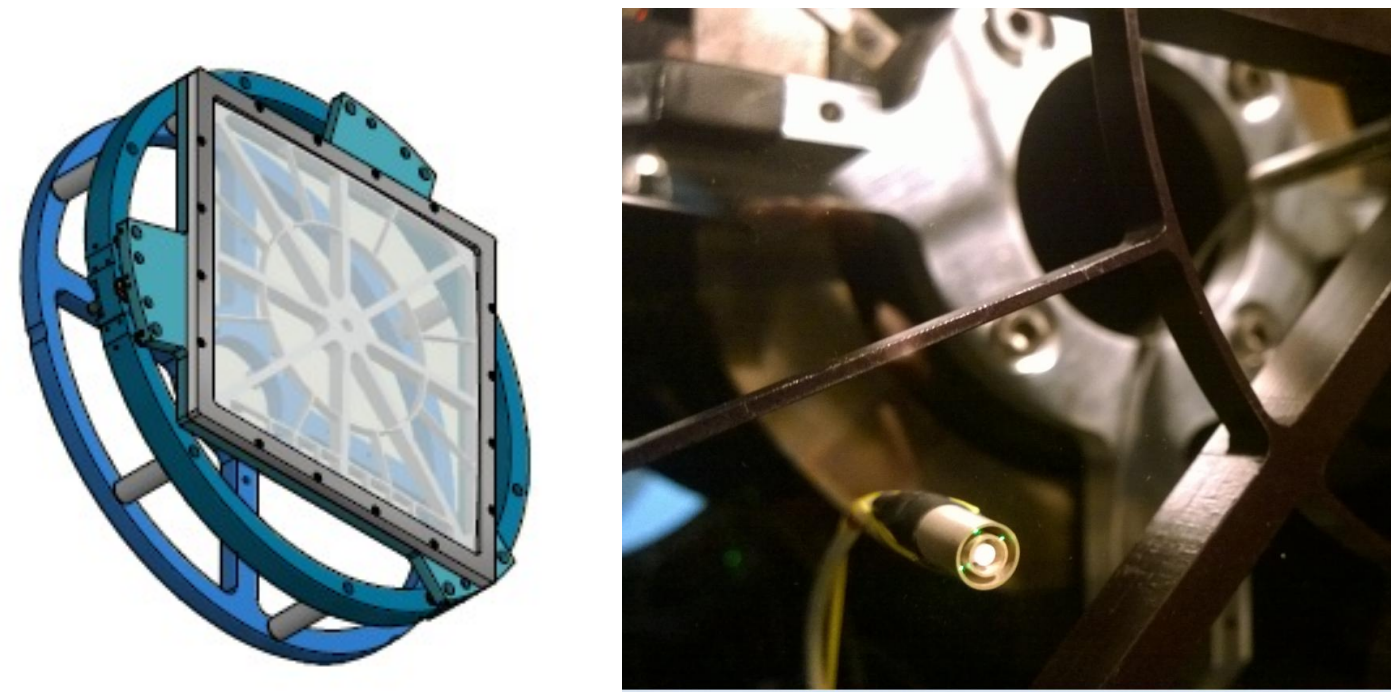

Figure 7. Left: Prototype field plate model for the on-sky experiments using Starbugs at the UKST. Right: Starbug mounted on the prototype field plate at the telescope.

\subsection{TAIPAN}

The aims of the TAIPAN facility [13] are to:

1. act as a proof-of-concept for the Starbugs positioning technology, proposed for use on the Giant Magellan Telescope, and

2. deliver a capability to carry out a comprehensive spectroscopic galaxy and stellar survey of the Southern Hemisphere using a refurbished UK Schmidt Telescope. 


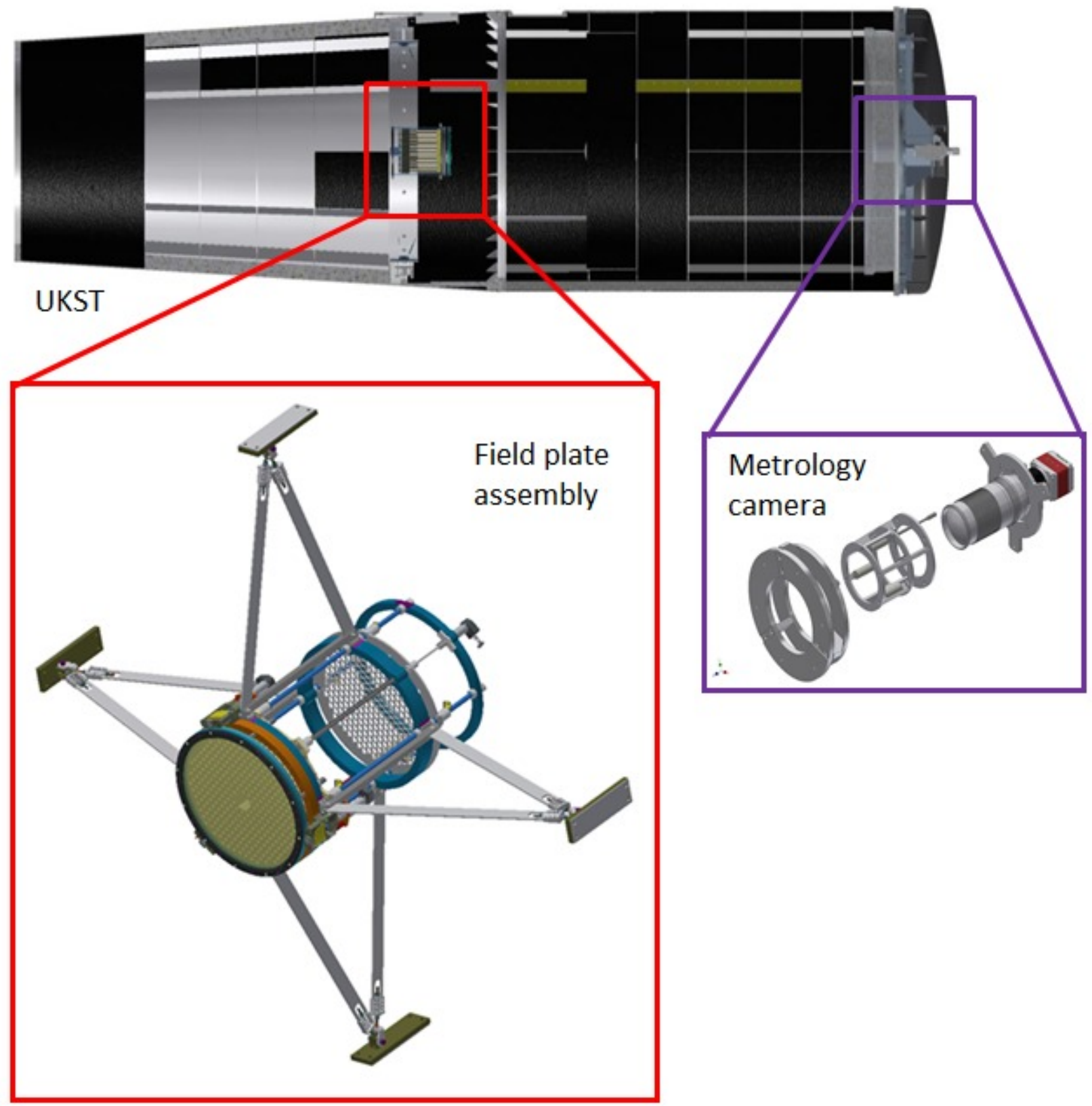

Figure 8. UKST telescope showing the TAIPAN field plate assembly (red box insert) that consists of the glass field plate mounted on a tip-tilt drive at the telescope focal plane in the centre of the telescope tube. As part of this assembly a 'bug catcher' is mounted immediately behind the glass field plate that can travel to the connector plate (shown unpopulated) and its associated motor drive. The metrology camera (purple box insert) consists of a high frame rate large format CCD with long focal length lens is mounted through the central hole in the primary mirror.

The TAIPAN instrument (see Figure 8) includes the MANIFEST prototype positioner and a new dedicated spectrograph currently being designed. The high level science requirements for this instrument are as follows:

- Simultaneous wavelength coverage: 370 to $870 \mathrm{~nm}$.

- Spectral resolution: $\mathrm{R}>2100$.

- Multiplex: 150 objects (upgradeable to 300 objects). 
- On-sky aperture per object: $3^{\prime \prime}$.

- Field of view: 6 degrees diameter.

- Extended object sensitivity: $\mathrm{r}=17$ for a Half-Light Radius=5" source in a 10 minute observation at $\mathrm{SNR}=1$ per resolution element in dark time.

- Point source sensitivity: $\mathrm{v}=11$ in a 1.5 minute observation for $\mathrm{SNR}=100$ per resolution element in bright time.

- Field reconfigure (including slew, configure, acquire, guide): 5 minutes (requirement), 2 minutes (goal).

- Cross talk between adjacent spectra: $4 \%$ (requirement), $2 \%$ (goal).

- Relative flux calibration: $10 \%$.

- Absolute wavelength calibration: $0.02 \mathrm{~nm}$ (requirement), $0.005 \mathrm{~nm}$ (goal).

\section{INSTRUMENT INTERFACES}

\subsection{G-CLEF}

The GMT-CfA, Carnegie, Catolica, Chicago Large Earth Finder (G-CLEF) is a fibre fed, optical echelle spectrograph that has been selected as one of the first light instruments on the GMT. G-CLEF [3] has been designed to be a generalpurpose echelle spectrograph with precision radial velocity capability. The spectrograph optical design is an asymmetric, two-arm, white pupil design, with a nominal beam size of $\sim 300 \mathrm{~mm}$, reduced to $200 \mathrm{~mm}$ after dispersion by the R4 echelle grating. The peak efficiency of the spectrograph is $>35 \%$ and the passband is $350-950 \mathrm{~nm}$. The spectrograph is fed with three sets of fibres to enable three observing modes (each for a single object plus sky): high-throughput ( $\mathrm{R} \sim 25 \mathrm{k})$, precision-abundance ( $\mathrm{R} \sim 40 \mathrm{k})$, and precision radial velocity $(\mathrm{R} \sim 120 \mathrm{k})$.

The addition of MANIFEST provides an important high multiplex mode for G-CLEF. We anticipate using a set of 43 single-fibre Starbug units (each 0.7" diameter) that feed a single slit within G-CLEF. With all but the central 4 probes' output masked on the spectrograph entrance slit, one gets multi-order spectra for 4 objects at once over the 370-950 nm range. With all 43 probes and a wedged Fabry-Perot filter, one gets a series of $\sim 7$ well-separated chunks of the spectrum, tunable in the sense that the whole series can be shifted up and down in wavelength. Any desired echelle order can be selected, along with sections of other orders. This gives a higher multiplex, but at the expense of spectral coverage.

The interface between MANIFEST and G-CLEF is currently being developed. It is likely that the MANIFEST fibres (and their associated post-optics) will be installed in the G-CLEF Dewar through a dedicated access panel in the Dewar wall, in such a way they do not disturb the standard G-CLEF slit. A mechanism external to the Dewar will allow light from unused fibres to be blocked in low-multiplex MANIFEST mode or for G-CLEF operation. A filter external to the Dewar (likely to be mechanised) will be used to prevent overlapping orders in the MANIFEST high multiplex mode.

\subsection{GMACS}

GMACS is a visible multi-object slit-mask spectrograph that covers a relatively wide field and uses wavelength multiplexing to deliver a large $A \Omega-\lambda$ combination [4]. Dichroic mirrors feed cameras optimized over single octave bandpasses. The full instrument will deliver moderate resolution $(R=1500-5000)$ spectroscopy from the near $U V$ to very near-IR regions of the spectrum.

MANIFEST brings significant benefits to the GMACS stand-alone modes. It provides improvement to multiplex via efficient detector packing and spectral resolution via image-slicing, and provides access to integral field unit modes. For the baseline design, two sets of fibre probes were envisioned. The first intended for survey type work, and in particular Lyman Alpha tomography of the intergalactic medium, comprised a set of 420, 19-fibre, 1.25" diameter field science or sky probes feeding the four GMACS slits. The probes are parked on a quasi-regular grid in the 20' diameter field. The second mode consisted of a single 8400-fibre integral field unit with a 23" diameter field feeding the four GMACS spectrographs. This non-moving probe is positioned near the field center. A silt unit would be used to select the operating mode (see Figure 9).

The GMACS team is now in the process of refining the concept for the GMACS instrument and considering trades between field of view, wavelength coverage, resolution, and operating modes. It is likely to be descoped in pixel count/field of view. This will provide even greater motivation for MANIFEST relative to the original baseline design. 
The revised GMACS may be designed for the bare Gregorian GMT focus, without a wide-field corrector. In this case the spectrograph design would no longer be pupil-centric, and some additional post-optics are required to feed it with fibers. This issue was already addressed in the MANIFEST feasibility study, for feeding the near infrared spectrograph NIRMOS [16]. A weak prism bonded to the flat final surface of each lenslet array restores pupilcentricity, with no significant loss of throughput. The prisms have a maximum thickness of just $3 \mathrm{~mm}$, so allow use with the slit mask.

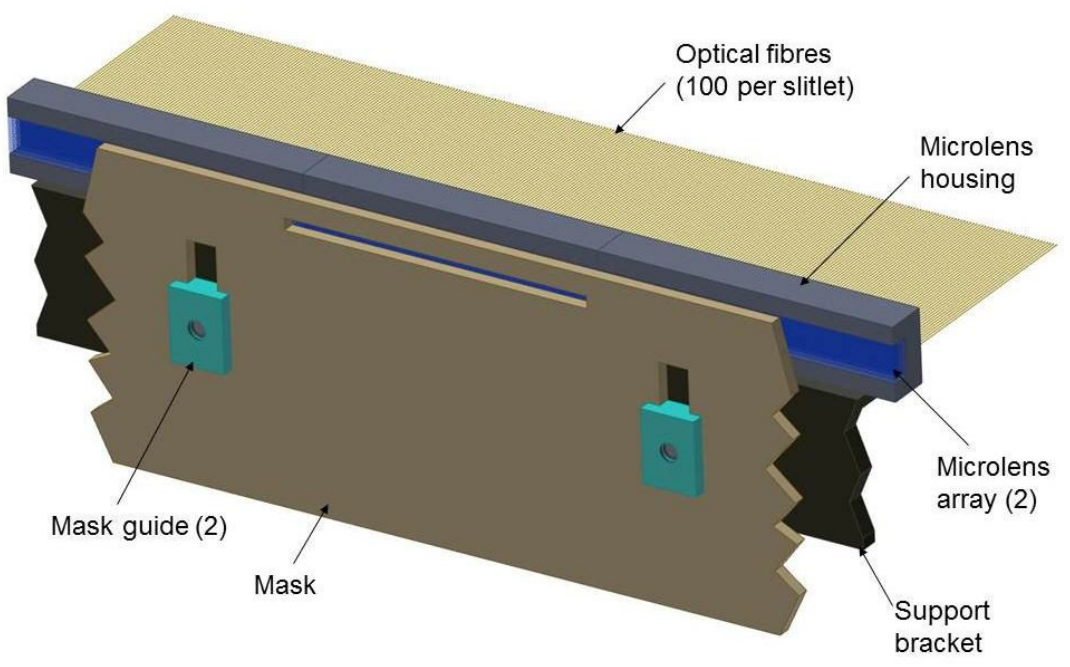

Figure 9. Initial concept for GMACS slit unit, showing mask used to select the fibre slit and allow nod and shuffle operation.

\subsection{Dedicated spectrographs}

Slit mask multi-object spectroscopic instruments inherently couple the optical design for the spectrograph to that of the telescope. For GMT, this necessitates a preference for large beam diameters, in particular, and fast cameras that are oversampled. An alternative, or potential additional upgrade, is to design the spectrographs explicitly to be fed via fibres. This has a key advantage in that the optical design of the spectrograph is no longer tightly coupled to the optical design of the telescope. It also allows the spectrographs to be made more modular, i.e., via image-slicing. We have been developing designs for a new modular optical spectrograph for the AAT for the Hector instrument concept $[14,15]$. In the next design phase we will consider the applicability of such spectrographs for use with MANIFEST on the GMT. We will also explore designs for near infrared spectrographs dedicated to be fibre fed from MANIFEST.

\section{SUMMARY}

MANIFEST is a fibre positioning system for the Giant Magellan Telescope that offers important gains for any seeing limited spectrograph. One of the key competitive advantages for GMT relative to the other Extremely Large Telescopes is that it has a relatively wide field of view. MANIFEST provides access to the full field of view available from the telescope design. It also provides a modular system that will allow for additional instrument feeds in the future.

\section{REFERENCES}

[1] Saunders, W., Colless, M., Saunders, I., Hopkins, A., Goodwin, M., Heijmans, J., Brzeski, J., and Farrell, T., "MANIFEST: a many-instrument fiber-positioning system for GMT," Proc. SPIE 7735, 773568 (2010).

[2] Goodwin, M., Brzeski, J., Case, S., Colless, M., Farrell, T., Gers, L., Gilbert, J., Heijmans, J., Hopkins, A., Lawrence, J., Miziarski, S., Monnet, G., Muller, R., Saunders, W., Smith, G., Tims, J., Waller, L., "MANIFEST instrument concept and related technologies," Proc. SPIE 8446, 84467I (2012).

[3] Szentgyorgyi, A., Frebel, A., Furesz, G., Hertz, E., Norton, T., Bean, J., Bergner, H., Crane, J., Evans, J., Evans, I., Gauron, T., Jordán, A., Park, S., Uomoto, A., Barnes, S., Davis, W., Eisenhower, M., Epps, H., Guzman, D., McCracken, K., Ordway, M., Plummer, D., Podgorski, W., Weaver, D., "The GMT-CfA, Carnegie, Catolica, 
Chicago Large Earth Finder (G-CLEF): A General Purpose Optical Echelle Spectrograph for the GMT with Precision Radial Velocity Capability," Proc. SPIE 8446, 84461H (2012).

[4] DePoy, D. L., Allen, R., Barkhouser, R., Boster, E., Carona, D., Harding, A., Hammond, R., Marshall, J. L., Orndorff, J., Papovich, C., Prochaska, K., Prochaska, T., Rheault, J. P., Smee, S., Shectman, S., Villanueva, S., "GMACS: a wide field, multi-object, moderate-resolution, optical spectrograph for the Giant Magellan Telescope," SPIE Proc 8446, 84461N (2012).

[5] McGrath, A., Moore, A., "Starbug: enabling the smart focal plane," Proc. SPIE 5495, 600-610 (2004).

[6] Goodwin, M., Heijmans, J., Saunders, I', Brzeski, J., Saunders, W., Mueller, R., Haynes, R., Gilbert, J., "Starbugs: focal plane fiber positioning technology," Proc. SPIE 7739, 77391E (2010).

[7] Gilbert, J., Goodwin, M., Heijmans, J., Mueller, R., Miziarski, S, Brzeski, J., Waller, L., Saunders, W., Bennet, A., Tims, J., "Starbugs: all-singing, all-dancing fibre positioning robots," Proc. SPIE 8450, 84501A (2012).

[8] Brown, D,. Case, S., Gilbert, J., Goodwin, M., Jacobs, D., Kuehn, K., Lawrence, J., Lorente, N., Nichani, V., Saunders, W., Staszak, N., Tims, J., "Starbug fibre positioning robots: performance and reliability enhancements," Proc SPIE 9151, in press (2014).

[9] Goodwin, M., Lorente, N. P. F., Hong, S. E., Satorre, C., Lawrence, J., "Field target allocation and routing algorithms for starbugs," Proc. SPIE 9152, in press (2014).

[10] Ellis, S. C., Ireland, M., Lawrence, J. S., Tims, J., Staszak, N., Brzeski, J., Parker, Q. A., Sharp, R., BlandHawthorn, J., Case, S., Colless, M., Croom, S., Couch, W., De Marco, O., Glazebrook, K., Saunders, W., Webster, R., Zucker, D., "KOALA: a wide-field 1000 element integral-field unit for the Anglo-Australian Telescope," Proc. SPIE 8446, 84460V (2012).

[11] Croom, S. M., Lawrence, J. S., Bland-Hawthorn, J., Bryant, J. J., Fogarty, L., Richards, S., Goodwin, M., Farrell, T., Miziarski, S., Heald, R., Jones, D. H., Lee, S., Colless, M., Brough, S., Hopkins, A. M., Bauer, A. E., Birchall, M. N., Ellis, S., Horton, A., Leon-Saval, S., Lewis, G., López-Sánchez, Á. R., Min, S., Trinh, C., Trowland, H., "The Sydney-AAO Multi-object Integral field spectrograph," MNRAS 421, 872 (2012).

[12] Ireland, M. J., Barnes, S., Cochrane, D., Colless, M., Connor, P., Horton, A., Gibson, S., Lawrence, J., Martell, S., McGregor, P., Nicolle, T., Nield, K., Orr, D., Robertson, J. G., Ryder, S., Sheinis, A., Smith, G., Staszak, N., Tims, J., Xavier, P., Young, P., Zheng, J., "The AAO's Gemini High-Resolution Optical SpecTrograph (GHOST) concept," Proc SPIE 8446, 844629 (2012).

[13] Kuehn, K., Lawrence, J. S., Brown, D., Case, S., Colless, M., Content, R., Gers, L., Goodwin, M., Hopkins, A., Ireland, M., Lorente, N., Muller, R., Nichani, V., Rakman, A., Saunders, W., Staszak, N., Tims, J., Waller, L., "TAIPAN: Optical Spectroscopy with StarBugs," Proc. SPIE 9147, in press (2014).

[14] Saunders, W., "A fast new cadioptric design for fiber-fed spectrographs," Proc SPIE 8446, 844654 (2012).

[15] Lawrence, J., Bland-Hawthorn, J., Bryant, J., Brzeski, J., Colless, M., Croom, S., Gers, L., Gilbert, J., Gillingham, P., Goodwin, M., Heijmans, J., Horton, A., Ireland, M., Miziarski, S., Saunders, W., Smith, G., "Hector: a high-multiplex survey instrument for spatially resolved galaxy spectroscopy," Proc SPIE 8446, 844653 (2012).

[16] Fabricant, D., Fata, R., Brown, W. R., McLeod, B., Mueller, M., Gauron, T., Roll, J., Bergner, H., Geary, J., Kradinov, V., Norton, T., Smith, M., Zajac, J., "NIRMOS: a wide-field near-infrared spectrograph for the Giant Magellan Telescope," Proc. SPIE 8446, 844610 (2012). 